\title{
A new analysis technique to measure fusion excitation functions with large beam energy dispersions
}

\author{
P.Figuera $^{1}$, A.Di Pietro ${ }^{1}$, M.Fisichella ${ }^{1}$, M.Lattuada ${ }^{1,2}$, A.C. Shotter $^{3}$, C. Ruiz ${ }^{4}$ and \\ M.Zadro ${ }^{5}$ \\ ${ }^{1}$ INFN Laboratori Nazionali del Sud, Catania, Italy \\ ${ }^{2}$ Dipartimento di Fisica ed Astronomia Universita' di Catania, Catania, Italy \\ ${ }^{3}$ University of Edinburgh, Edinburgh, UK \\ ${ }^{4}$ TRIUMF, Vancouver, Canada \\ ${ }^{5}$ Ruder Boskovic Institute, Zagreb, Croatia
}

\begin{abstract}
Peculiar nuclear structures of two colliding nuclei such has clustering, neutron halo/skin or very low breakup thresholds can affect the reaction dynamics below the Coulomb barrier and this may also have astrophysical consequences. In order to have a better understanding of this topic, in the last decade, several experiments were performed. A typical experimental challenge of such studies is the need to measure excitation functions below the Coulomb barrier, having a strong energy dependence, with rather large beam energy dispersions inside the target. This may easily lead to ambiguities in associating the measured cross section with a proper beam energy. In this paper a discussion on this topic is reported and a new technique to deal with the above problem will be proposed.
\end{abstract}

\section{Introduction}

In the last decade many efforts have been devoted to a better understanding of structure effects on reaction dynamics below the Coulomb barrier (see e.g. [1] and references therein). Besides being a challenging topic in itself, both from an experimental and theoretical point of view, a better understanding of structure effects on the dynamics can also improve the quality of calculations in certain astrophysical scenarios. As an example, one of the uncertainties when attempting to calculate fusion of extremely neutron rich ions in X-ray superbursts is the poorly known effect of the neutron skin on the dynamics e.g. [2]. It has also been recently suggested that clustering affects the dynamics below the barrier influencing the extraction of the electron screening potential [3] and a better understanding of such effects can shed some light on the so called 'electron screening puzzle'.

A typical experimental challenge of such studies, often performed with low intensity radioactive beams (RIBs), is the need to measure excitation functions below the barrier, having a strong energy dependence, with rather large beam energy dispersions inside the target due to a combination of target thickness/uniformity and incoming beam energy 
spread. This problem usually becomes very important when measuring cross sections with activation techniques irradiating stacks of several targets, which is a common procedure when using low intensity RIBs (see e.g. [4] and references therein). The beam energy spread inside the target may easily lead to ambiguities in associating the measured cross section to a proper beam energy. In this paper a discussion on this topic is reported and a new technique to deal with the above problem will be discussed.

\section{Unfolding procedures}

Many fusion reactions induced by low intensity RIBs around and below the barrier have been studied by irradiating stacks of several thick targets and measuring on-line or off-line the radiation emitted in the decay of the evaporation residues. As discussed in [4], in some of these experiments the authors had very large beam energy distributions inside the targets in the range 2-6 MeV FWHM. In these activation experiments, the fusion excitation function has been determined by associating the cross-section $\sigma_{\text {mean }}$, measured in each target, either with the average energy in the considered target $\bar{E}$ (see e.g. [5,6]) or with an effective energy $E_{\text {eff }}$, (see e.g. [7,8] ) defined as follows:

$$
E_{\text {eff }}=\frac{\int_{0}^{\infty} E \sigma(E) D\left(E, t_{0}\right) d E}{\int_{0}^{\infty} \sigma(E) D\left(E, t_{0}\right) d E}
$$

where $D\left(E, t_{0}\right)$ represents the probability that a beam particle will have energy $\mathrm{E}$ inside the considered target of thickness $t_{0}$. This has been calculated with different degrees of approximation by various authors. However, it can be shown already analytically [4] that the above methods of relating $\sigma_{\text {mean }}$ to $\bar{E}$ or to $E_{\text {eff }}$ can result in misinterpretation of the real excitation function. As an example, in figure 1, the result of a numerical simulation concerning the study of the ${ }^{9} \mathrm{Li}+{ }^{120} \mathrm{Sn}$ fusion reaction is reported [4]. It has been supposed that a $28 \mathrm{MeV}^{9} \mathrm{Li}$ beam impinged on a stack of five targets each composed of one ${ }^{120} \mathrm{Sn}$ foil with an average thickness of $5 \mathrm{mg} / \mathrm{cm}^{2}$, followed by a ${ }^{93} \mathrm{Nb}$ catcher/degrader foil 1.5 $\mathrm{mg} / \mathrm{cm}^{2}$ thick. The simulations were performed for the case of uniform and non uniform targets and degrader foils. The ${ }^{9} \mathrm{Li}+{ }^{120} \mathrm{Sn}$ fusion excitation function has been supposed to be known and to have a Wong-like [10] behavior with realistic parameters, resulting in the black line of figure 1 . The beam energy distributions inside the targets $D\left(E, t_{0}\right)$ have been calculated exactly with the help of the SRIM code [9] as discussed in [4]. In this way, for each target, the mean cross-section that would be measured in it has been calculated by using the following relation:

$$
\sigma_{\text {mean }}=\frac{\int_{0}^{\infty} \sigma(E) D\left(E, t_{0}\right) d E}{\int_{0}^{\infty} D\left(E, t_{0}\right) d E}
$$



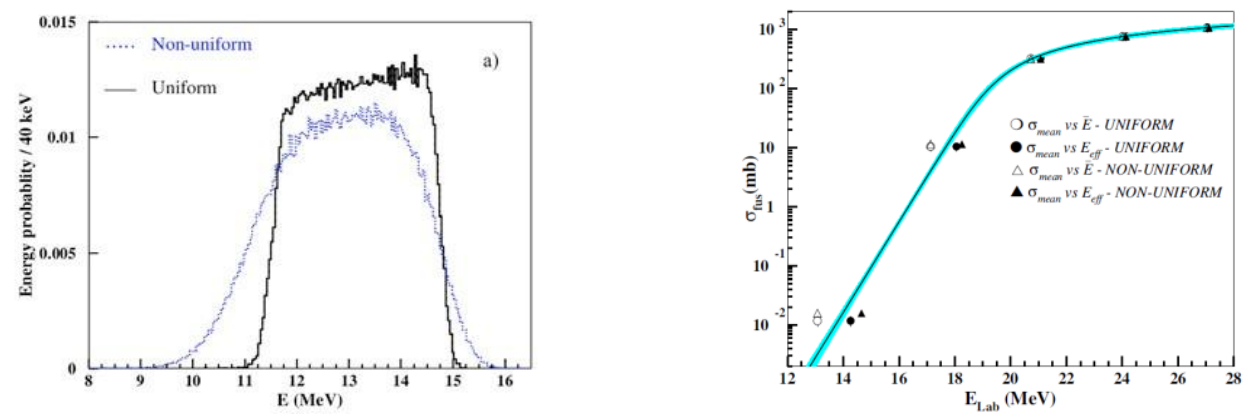

Fig. 1. Results of the simulations for the study of the ${ }^{9} \mathrm{Li}+{ }^{120} \mathrm{Sn}$ fusion reaction. (a) Beam energy probability distribution $\mathrm{D}\left(\mathrm{E}, \mathrm{t}_{0}\right)$ inside the last target of the stack assuming uniform and non uniform foils. (b) The continuous black line shows the real fusion excitation function assumed for the collision. The open and closed symbols are the experimental results that would be obtained plotting the measured $\sigma_{\text {mean }}$ versus $\bar{E}$ and $E_{\text {eff }}$ (circles uniform foils triangles non uniform foils). The blue shaded area represents the result of our deconvolution procedure with its associated uncertainty. See text for details.

In Fig. 1 the $\sigma_{\text {mean }}$ obtained by applying Eq. (2) is plotted versus the mean energy $\bar{E}$ (open symbols), and versus the effective energy $E_{\text {eff }}$ (closed symbols). As one can see, at energies below the Coulomb barrier, the real excitation function (black line) is not reproduced by any of the two representations. Plotting the measured cross-sections versus $\bar{E}$ or $E_{\text {eff }}$ generates differences up to a factor of 5 with respect to the real fusion excitation function.

As a possible alternative solution, we propose the following unfolding procedure. The task is to deduce the continuous excitation function $\sigma(E)$ from a finite number of cross sections $\sigma_{\text {mean }} i$ measured in the considered experiment. This may be done by choosing a suitable function $\hat{g}(E, \mu)$ (with $\mu$ parameters to be determined) which shows the same expected energy behavior as $\sigma(E)$, and then minimizing the following expression $\mathrm{S}$ with respect to $\mu$ :

$$
S=\sum_{i}\left(\frac{\sigma_{\text {mean }, i}-\hat{g}_{\text {mean }, i}}{\beta_{i}}\right)^{2} \quad \text { where } \quad \hat{g}_{\text {mean }, i}=\frac{\int_{0}^{\infty} \hat{g}(E, \mu) D\left(E, t_{0}\right) d E}{\int_{0}^{\infty} D\left(E, t_{0}\right) d E}
$$

Here $\beta_{\mathrm{i}}$ is the experimental error associated with $\sigma_{\text {mean }}, i$. Thus the goal of the proposed deconvolution procedure is the deduction of the best curve $\hat{g}(E, \mu)$ which, when convoluted with the $D\left(E, t_{0}\right)$ functions, for each target, reproduces the value of the experimental mean cross sections. The blue shaded area in figure 1 represents the result of the above unfolding procedure applied using a 3 parameter Wong-like function [10] as $\widehat{g}(E, \mu)$ and its error.

A problem in the suggested approach is that, given a functional form, it will always be possible (from a mathematical point of view) to apply the proposed procedure and obtain a deconvoluted excitation function, which may differ from the one obtained plotting the 
measured cross sections as a function of the mean energy. It is therefore important to check if the above-mentioned differences are simply due to the fact that the chosen functional form is not able to reproduce the real excitation function, or the differences are, instead, to be attributed to the real effect of the energy spread of the beam. A self consistency check that can be done, to verify if the chosen $\hat{g}(E, \mu)$ is a good representation of the fusion excitation function, is the following. One can calculate the ratio between the $\sigma_{\text {calc-mean,i, }}$ obtained from Eq. (2) averaging the best functional form $\hat{g}(E, \mu)$ (deduced by the deconvolution procedure) over the beam energy distribution in the target $\mathrm{D}_{\mathrm{i}}\left(\mathrm{E}, \mathrm{t}_{0}\right)$, and the measured cross section $\sigma_{\text {mean,i. }}$. If this ratio is equal to 1 within the error in the whole energy range, then the procedure is self consistent. As an example, we recently applied this approach in [11].

In our study of the above discussed problems, we also verified that another way to extract a correct excitation function when dealing with large beam energy dispersion is the recursive procedure suggested in [12]. This is true, however, only if the correct $\mathrm{D}\left(\mathrm{E}, \mathrm{t}_{0}\right)$ is used in the procedure, whereas in [12] the authors use an approximation, taking only into account the effects of the energy loss.

\section{Summary and conclusions}

In the present study we have shown that the traditional way to represent an experimental fusion cross section, as a function of the average energy inside the target or as a function of an effective energy based on a weighted average, may lead to a wrong determination of the excitation function in the case of large beam energy dispersions and an alternative unfolding procedure was proposed and discussed. We believe that, in future publications where such kind of data are presented, a complete discussion on the beam energy spread and its effects on the deduced excitation functions should be presented.

\section{References}

1. L.F. Canto et al., Phys Rep. 596, 1, (2015) and references therein

2. C.J. Horowitz et al., Phys. Rev. C 77, 045807, (2008)

3. C. Spitaleri et al., Phys. Lett. B 755, 275, (2016)

4. M. Fisichella et al., Phys. Rev. C 92, 064611, (2015)

5. Y.E. Penionzhkevich et al., Phys. Rev. Lett. 96, 162701, (2006)

6. Y.E. Penionzhkevich et al., Eur. Phys. Jour. A 31, 185, (2007)

7. R. Wolksi et al., Eur. Phys. Jour. A 47, 111, (2011)

8. D. Shapira et al. Nucl. Instr. and Meth. A 551, 330, (2005)

9. J.F. Ziegler, 'SRIM code' www.srim.org

10. C. Y. Wong, Phys. Rev. Lett. 31, 766, (1973)

11. M. Fisichella et al., Phys Rev. C 95, 034617, (2017)

12. J.F. Liang et al. Phys. Rev. C 75, 054607, (2007) 